Recent Developments in Dynamic Transmission Electron Microscopy

N. D. Browning, M. A. Bonds, G. H. Campbell, J. E. Evans, T. LaGrange, K. L. Jungjohann, D. J. Masiel, J. McKeown, S. Mehraen, B. W. Reed, M. Santala

April 20, 2011

Current Opinion in Solid State and Materials Science 
This document was prepared as an account of work sponsored by an agency of the United States government. Neither the United States government nor Lawrence Livermore National Security, LLC, nor any of their employees makes any warranty, expressed or implied, or assumes any legal liability or responsibility for the accuracy, completeness, or usefulness of any information, apparatus, product, or process disclosed, or represents that its use would not infringe privately owned rights. Reference herein to any specific commercial product, process, or service by trade name, trademark, manufacturer, or otherwise does not necessarily constitute or imply its endorsement, recommendation, or favoring by the United States government or Lawrence Livermore National Security, LLC. The views and opinions of authors expressed herein do not necessarily state or reflect those of the United States government or Lawrence Livermore National Security, LLC, and shall not be used for advertising or product endorsement purposes. 


\title{
Recent Developments in Dynamic Transmission Electron Microscopy
}

\author{
N. D. Browning ${ }^{1,2,3}$, M. A. Bonds ${ }^{1}$, G. H. Campbell ${ }^{3}$, J. E. Evans ${ }^{2}$, T. LaGrange ${ }^{3}$, \\ K. L. Jungjohann ${ }^{1}$, D. J. Masiel ${ }^{1}$, J. McKeown ${ }^{3}$, S. Mehraeen ${ }^{2}$, B. W. Reed ${ }^{3}$, M. Santala ${ }^{3}$ \\ ${ }^{1}$ Department of Chemical Engineering and Materials Science, University of California - Davis, \\ One Shields Ave, Davis, CA 95616. \\ ${ }^{2}$ Department of Molecular and Cellular Biology, University of California - Davis, One Shields \\ Ave, Davis, CA 95616. \\ ${ }^{3}$ Condensed Matter and Materials Division, Physical and Life Sciences Directorate, Lawrence \\ Livermore National Laboratory, Livermore, CA 94550.
}

\begin{abstract}
One of the current major driving forces behind instrument development in transmission electron microscopy (TEM) is the ability to observe materials processes as they occur in-situ within the microscope. For many processes, such as nucleation and growth, phase transformations and mechanical response under extreme conditions, the beam current in even the most advanced field emission TEM is insufficient to acquire images with the temporal resolution ( $1 \mu \mathrm{s}-1 \mathrm{~ns})$ needed to observe the fundamental interactions taking place. The dynamic transmission electron microscope (DTEM) avoids this problem by using a short pulse laser to create an electron pulse of the required duration through photoemission which contains enough electrons to form a complete high resolution image. The current state-of-theart in high time resolution electron microscopy in this paper describes the development of the electron optics and detection schemes necessary to fully utilize these electron pulses for materials science. In addition, developments for future instrumentation and the experiments that are expected to be realized shortly will also be discussed.
\end{abstract}

Keywords: Dynamic TEM, Dark-field imaging, In-situ microscopy, liquid stages, gas stages 


\section{Introduction}

Transmission electron microscopy (TEM) has long played a key role in driving our scientific understanding of extended defects and their control of the properties of materials. The current generation of aberration corrected and monochromated TEMs, can now obtain unprecedented spatial resolution (approaching $0.05 \mathrm{~nm}$ ) in both images and electron energy loss spectra (EELS) [1-5]. However, for all of the high spatial resolution possible in these new microscopes, a key feature of all the experiments is that the object being studied has to remain stationary. Typically there is a constant battle just to overcome drift, charging, mechanical instabilities, stray fields, beam damage etc, to achieve the required stability criterion - and thus the whole approach is aimed more at stability than achieving the flexibility to study dynamic events.

Dynamic events have been studied in a TEM before, with a whole sub-field of in-situ microscopy studying the effects of mechanical deformation, the effect of gas pressure on catalytic activity and nanostructure nucleation and growth, beam damage and even reactions taking place in liquids [6-15]. In all of these cases, spatial resolution is sacrificed for either the in-situ conditions and/or to achieve temporal resolution. For the typical beam currents in commercial thermionic and field emission electron microscopes, a practical limit of $1 \mathrm{~ms}$ temporal resolution is defined simply by the limit in the number of electrons that reach the detector in that time interval. In many cases, this means that the imaging process itself is just too slow to see the critical details of the phenomenon being studied - it may as well just be a static measurements.

There is, however, a wide range of dynamic phenomena that occur in both inorganic and organic structures on timescales well below $1 \mathrm{~ms}$. In some cases, as with dislocation motion, it is not necessary to achieve atomic spatial resolution to observe the phenomenon, while in others, such as atomic diffusion, it is. There is therefore a range of length and time scales that it would be ideal to access by experimental techniques - roughly 1 micron to 1 angstrom spatial resolution coupled with 1 microsecond to 1 femtosecond temporal resolution. This desire to achieve high temporal/spatial resolution is not new, and dynamic observations have been shown previously by optical and X-ray means [16-18]. However, while femtosecond spectroscopy and pump-probe experiments have proved to be very successful, they are typically limited in spatial resolution and often require the image to be inverted from a diffraction pattern - there is no direct image of the process taking place. These limitations can be overcome by using electron pulses on the same timescale to analyze materials [19]. The immediate benefit of using electrons is that the interaction of the electrons with the material being analyzed is much stronger, producing more signal. This advantage has been used for many years in the field of ultrafast electron diffraction (UED) [20-23]. If the electron pulses can now be combined with the TEM methods mentioned above, then the beam can be directed and focused, images, spectra and diffraction patterns can be obtained from localized areas and direct high resolution images of dynamic events can also be obtained, thereby avoiding the need to invert the diffraction pattern.

The ability to study dynamic processes in materials on a timescale approaching 1 nanosecond is the main driving force behind the development of the dynamic transmission electron microscope (DTEM) at Lawrence Livermore National Laboratory (LLNL) [24-30]. To achieve this temporal resolution while still maintaining the direct high resolution imaging 
capability of a TEM, required the modification of a conventional TEM to create and control large electron bunches (containing $\sim 10^{9}$ electrons) - this development follows the groundbreaking research of Bostanjoglo and co-workers in this area [31-33]. In this paper, the basic physical principles behind the creation of electron bunches, their control and the expected TEM image resolution are defined. The electron optics necessary to turn a conventional TEM into a DTEM are described for both the current DTEM and for potential new instruments that are on the horizon. It should be noted here that the goal of this particular development is to obtain complete images from single shot experiments, making the approach radically different from the ultrafast, or stroboscopic, TEM that has been developed by Zewail and co-workers [34,35].

\section{The Physics of Short Electron Pulses in TEM}

The engineering of a single-shot DTEM starts with a physical understanding of the electron pulses themselves [36-38]. To achieve the time resolution, the DTEM has to operate at extremely high current densities ( $10^{9}$ electrons per pulse) with minimal sacrifice of spatial and temporal coherence - which ensures that the microscope can still form images. Perhaps most critical for the imaging process in DTEM, is that large local convergence angles $\alpha$ at the sample plane (which are equivalent to small spatial coherence lengths) shown in Figure 1(a)) tend to wash out the contrast. This is important because $\alpha$ plays a central role in the law of conservation of brightness (discussed below); increasing current density at the sample generally comes at the cost of increasing $\alpha$ and thus reducing the spatial coherence.

The range of acceptable convergence angles may vary greatly with the contrast mechanism and spatial frequency range of interest. But no matter the mechanism, a reduction in spatial coherence eventually reduces the relevant image contrast to the point where the features of interest are indistinguishable from artifacts and noise - a textbook example is the way in which the convergence angle cuts off the contrast-transfer function for high-resolution phase-contrast imaging. It should also be noted that temporal incoherence (usually expressed in terms of energy spread), by coupling to the lens system's chromatic aberration, also reduces contrast in a similar way and must be kept to a minimum.

Single-shot DTEM adds an additional constraint to the conventional imaging modes in TEM namely that a single nanosecond-scale pulse contain enough electrons to provide an image of acceptable contrast and resolution. This eliminates the option of simply increasing spatial coherence by introducing apertures and adjusting condenser lenses and acquiring for a longer time. It also puts stringent requirements on the brightness and current of the electron pulse the brightness must be at least $\sim 10^{7} \mathrm{~A} \cdot \mathrm{cm}^{-2} \cdot$ steradian $^{-1}$ (and preferably orders of magnitude higher), while the beam current must be on the milliampere scale [38]. Add to this the requirement that the energy spread be kept below $5 \mathrm{eV}$, and this greatly restricts the kinds of electron sources that may be used - namely linear photoemission produced by ultraviolet pulsed lasers directed onto large-area metallic cathodes (although other sources are currently under development).

Besides these conventional effects, DTEM must contend with additional nonlinear effects specifically related to the extremely high current densities. This arises from one basic physical problem: electrons are charged Fermions. Thus they will repel each other over long distances, 
collide with each other at short distances, and refuse to fill phase space (the 6-dimensional space combining three-dimensional position with three-dimensional momentum) more densely than one electron per quantum state. As a result, electron beams are subject to space charge effects, inhomogeneous scattering effects, and fundamental limits on spectral brightness (i.e. brightness for a given energy spread). Space charge effects limit the current density extractable from an electron gun geometry and (together with the energy spread) are an important factor in determining the maximum brightness attainable with a given gun. They also create lens aberrations and defocus electron beams (forcing slight adjustments of lens strengths as the beam current changes).

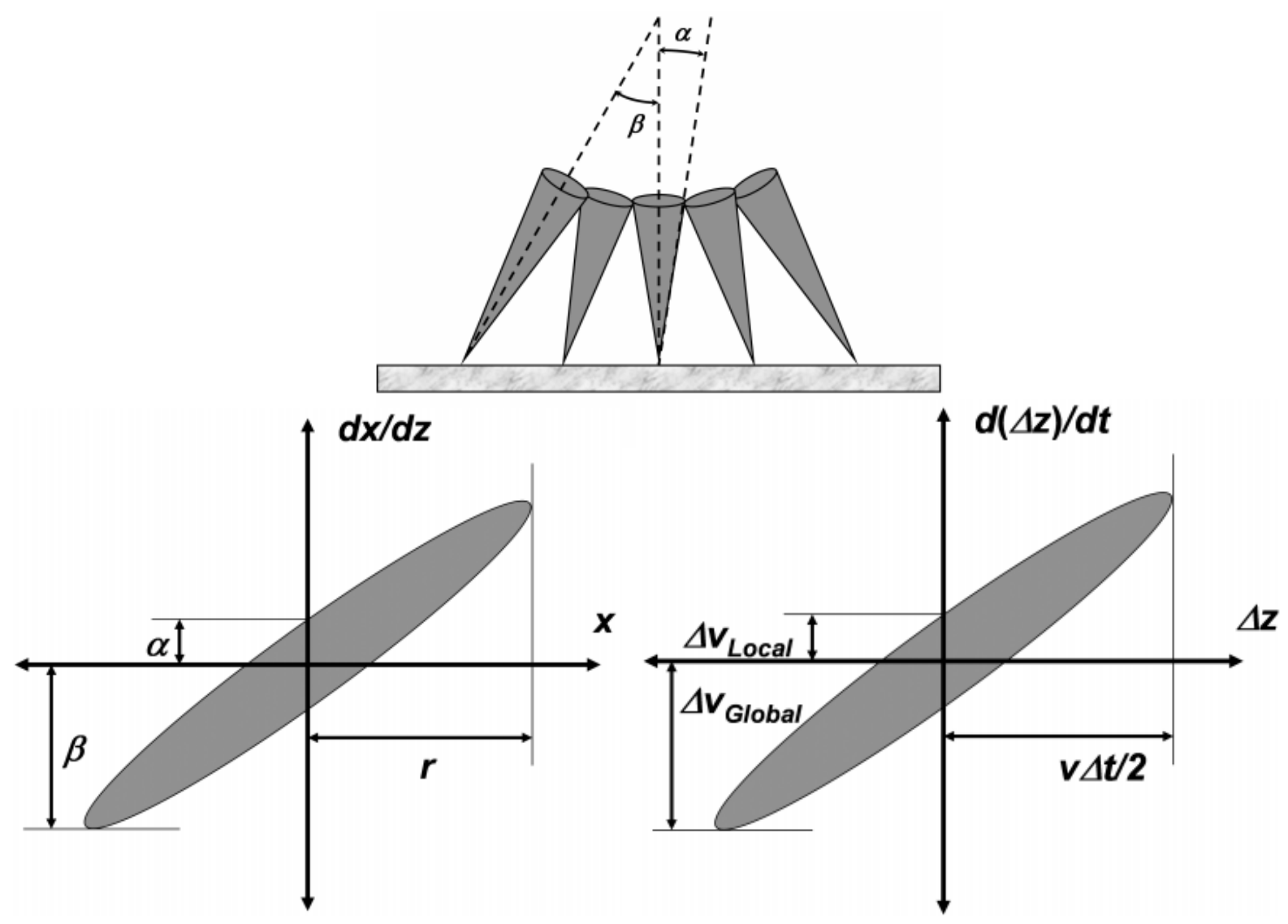

Figure 1: (a) Illustrating local $(\alpha)$ versus global $(\beta)$ measures of beam convergence/divergence. While $\alpha$ is a measure of lateral spatial coherence at a given point, and the product $\varepsilon=r \alpha$ is a nearly conserved quantity in the absence of apertures, $\beta$ can be changed rather arbitrarily if enough transfer lenses are available. (b) The same beam profile as a lateral phase-space ellipse. The emittance $\varepsilon$ is equal to the area of this ellipse times a factor of order unity. (c) A similar phase-space plot for the longitudinal $(z)$ direction, where $\Delta z$ is the axial distance of an electron from the pulse center of mass and $v$ is the nominal pulse velocity. In this case $\Delta v_{G l o b a l}$ is very important, as it couples to the chromatic aberration of the objective lens.

Ultimately, the performance of single-shot DTEM is governed by the brightness of the electron source. While this is true for any TEM, it is especially true for a single-shot DTEM 
which demands a very high fluence (electrons per unit area) be delivered in a very short time. We will define brightness $B$ as

$$
B=\frac{N e}{\left(\pi r^{2}\right)\left(\pi \alpha^{2}\right) \Delta t},
$$

where $N$ is the number of electrons per pulse, $e$ is the electronic charge, $r$ is the radius of the electron beam, $\alpha$ is the local convergence semi-angle, and $\Delta t$ is the time duration of a pulse. $B$ is very nearly conserved for a beam propagating at a fixed accelerating voltage. By "nearly conserved," we mean that $B$ is a constant in an ideal electron column, but in real systems a number of physical effects (e.g. Boersch effects, aberrations, and space charge effects) can cause the effective brightness to degrade with propagation distance. If the voltage is not constant, then the normalized brightness (equal to fundamental constants times $\lambda^{2} B$, with $\lambda$ the electron wavelength (using the relativistic deBroglie relation $\lambda=h /(\beta \gamma m c)$ )) is the relevant conserved quantity that includes all relativistic corrections. The quantities $r$, $\alpha$, and $\Delta t$ are finite and the brightness as we have defined it is an effective average over a finite area.

We also define the related quantity emittance as $\varepsilon=r \alpha$, which is itself a nearly-conserved quantity just as $B$ is, provided no electrons are blocked at apertures. A smaller $\varepsilon$ implies a higher-quality beam, with higher spatial coherence for a given spot size. The phase-space filled by electrons (Figure 1 b) can be transformed by lenses, so long as its area doesn't change. If the lenses are aberration-free, then a phase-space ellipse remains an ellipse throughout the column, even though its tilt and aspect ratio can change. Multipole lens elements such as those in aberration correctors can perform more complex operations, for example turning an aberrated sigmoidal shape into an approximate ellipse. In principle, a sufficiently complex lens system could allocate this phase-space area in just about any desired manner. In time-resolved electron microscopy, we also need to consider the longitudinal phase space (Figure 1c), which shows the distribution of electron speeds (or energies) as a function of arrival time (or of longitudinal position) relative to the center of mass of the bunch. The combination of this longitudinal phase space with the transverse phase spaces in the $x$ and $y$ directions (Fig. 1b) comprise the six-dimensional phase space mentioned above. Longitudinal phase space area is also approximately conserved and can be manipulated with various combinations of spacecharge expansion, ballistic propagation, and pulse compressors (which we may think of as temporal lenses).

The longitudinal space charge effects that cause $\Delta t$ to increase as a pulse propagates are only very weakly coupled to the aberrations that cause the emittance $\varepsilon$ to degrade (although they do affect the rate of evolution of the lateral phase space ellipse). Thus the product $B \Delta t$ (which is proportional to the number of electrons in a pulse) is also nearly conserved. Combining this effect with the variation of brightness with accelerating voltage, we find that the dimensionless quantity

$$
N_{C} \equiv \frac{\pi^{2} \lambda^{2} B \Delta t}{e}
$$

(which we will call the coherent fluence) is a convenient figure of merit that is very nearly constant as a function of propagation distance for any electron pulse we would likely be using. 
This definition is motivated by a recognition that the lateral coherence length is given by $r_{C}=$ $\lambda / \alpha$, so that our quantity $N_{C}$ is essentially the number of electrons per lateral coherence area, per pulse (to within definition-dependent factors of order unity). No amount of lensing, aperturing, acceleration, or space charge dynamics will allow a user to significantly improve this value once the pulse has left the gun. As we shall show, the coherent fluence plays a central role in the theory of resolution limits for single-shot pulsed imaging. For example, if $N_{C}$ is not much more than 1, then coherent single-shot imaging is impossible, no matter how good the lens system is.

To determine the optimum imaging conditions, we will consider the interplay of brightness, coherence, shot noise, and the instrumental contrast transfer function, with the goal of estimating the spatial resolution limit as a function of time resolution $\Delta t$. Here we use standard textbook formulae for a conventional TEM's partially coherent contrast transfer functions $(T(r))$ for both phase and amplitude contrast [39], including spatial and temporal incoherence effects through the usual envelope approximations. We will assume that the user is interested in getting the best possible contrast at a given spatial frequency, with a fixed brightness and single-shot time resolution, which means (1) adjusting the objective lens defocus to maximize $|T|$ for the desired spatial frequency band (this is done implicitly for all the results shown here), and (2) increasing the current density by converging the beam until $\alpha$ is large enough that the spatial incoherence is starting to significantly reduce $|T|$. We define a coherence factor $f_{\text {coherence }}=\lambda / r \alpha$, with $\lambda$ the electron wavelength, $r$ the radius of the smallest feature to be resolved, and $\alpha$ the half-width of the angular distribution function. In other words, we have scaled the pixel size to the lateral coherence length via the dimensionless factor $f_{\text {coherence }}$. Employing the above definition of brightness, we find that the number of electrons per pixel area $\pi r^{2}$ is

$$
N=\frac{\pi^{2} \lambda^{2} B \Delta t}{e} \cdot \frac{1}{f_{\text {coherence }}^{2}}=\frac{N_{C}}{f_{\text {coherence }}^{2}} \text {. }
$$

The coherent fluence has come up naturally from the imposition of our condition (2). We then apply the Rose criterion [40], which specifies the minimum number of particles that need to be detected before a pixel can be said to be resolved in the presence of shot noise. The governing formula is $N^{-1 / 2}=|T| / f_{\text {Rose, }}$, with $f_{\text {Rose }}$ typically set to $\sim 5$ and $T$ the contrast transfer function. Combining equations yields the formula

$$
N_{C}=\left(\frac{f_{\text {coherence }} f_{\text {Rose }}}{T}\right)^{2} \text {, }
$$

where the coherent fluence is set equal to a combination of dimensionless parameters. This defines a curve (actually two curves, one for phase and one for amplitude contrast, which have different values of $T(r)$ ) which can be plotted to show the tradeoff of spatial and temporal resolution (Figure 2 ). The absolute contrast transfer function $|T|$ is maximized with respect to defocus at the spatial frequency of one cycle per $2 r$ (where $r$ is the spatial resolution). We also sought to minimize the required $N_{C}$ as a function of $\alpha$, but the inverse-linear dependence of $f_{\text {coherence }}$ on $\alpha$ more than balanced the relatively weak $\alpha$ dependence of our approximate transfer function $T$, suggesting that the optimum was at extremely large convergence angles. 
However, the standard formulae for the spatial coherence envelope come from a first-order approximation, and they break down at large $\alpha$, so that the experimentally-relevant optimum is probably closer to $f_{\text {conerence }}=1$. In concrete terms, converging the electron beam increases the signal-to-noise ratio very quickly, but after a certain point the spatial incoherence rapidly gets so bad (in a way that isn't captured by the basic equations) that no imaging is possible. As a practical compromise, we match the coherence length to the target resolution, i.e. we set $f_{\text {coherence }}$ to 1 , for all coherent imaging modes.

Figure 2 also includes a simpler curve that estimates the resolution limit for incoherent high-angle annular dark field (HAADF) TEM imaging. Since this is an incoherent imaging mode, we no longer vary $\alpha$; rather we set it to a fairly large value (10 milliradians) which is on the order of the inner radius of an HAADF-TEM aperture [24]. The relevant formula becomes

$$
N_{C}=\left(\frac{\lambda f_{\text {Rose }}}{r \alpha}\right)^{2} f_{\text {collected }}^{-1} \text {, }
$$

where $f_{\text {collected }}$ is the fraction of electrons that scatter into the annular hole in the HAADFTEM aperture. This quantity varies a great deal with the aperture size and the mass-thickness of the sample; we have arbitrarily set it to 0.2 for purposes of rough calculation. The resolution in HAADF-TEM mode will be limited by spherical aberration to $r_{\min } \sim C_{s} \alpha^{3}$, and we have cut off the spatial resolution below this point for the HAADF curves in Figure 2.

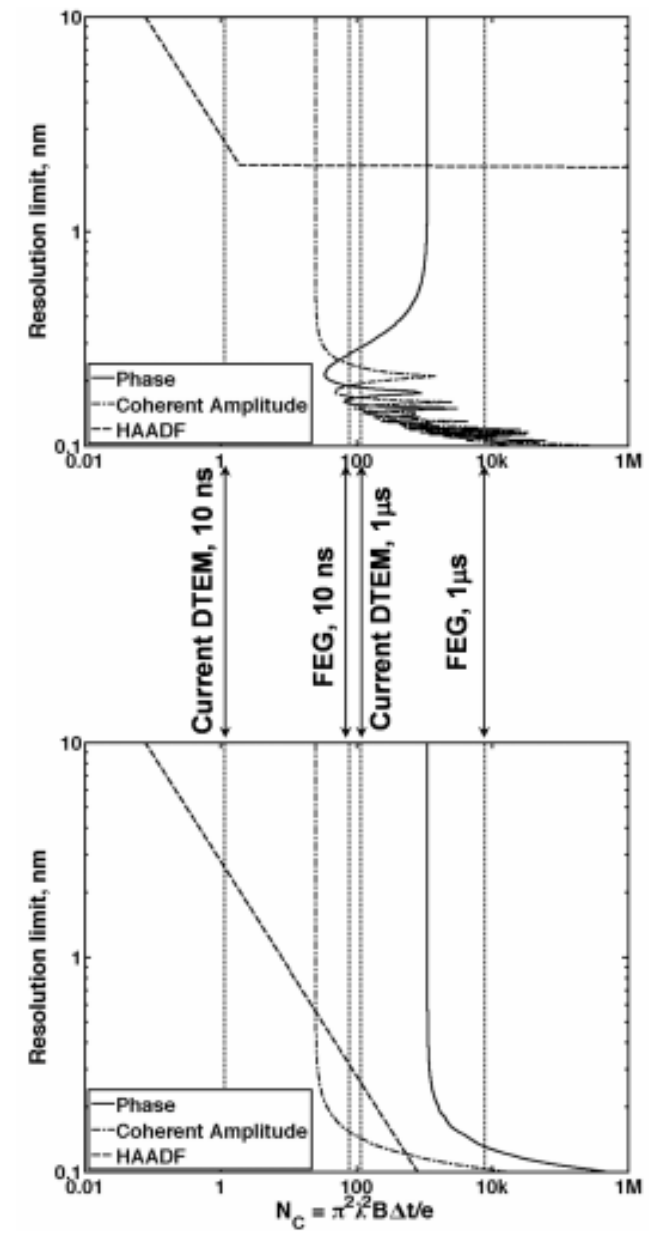

Figure 2: (Top) $\mathrm{C}_{s}$-limited and (Bottom) $\mathrm{C}_{s^{-}}$ corrected resolution limits as a function of the scaled product of brightness and pulse duration (or coherent fluence) for single-shot imaging. Vertical dashed lines are $N_{C}$ values for four scenarios, as indicated. Parameters are: $200 \mathrm{keV}$ kinetic energy, $\mathrm{C}_{\mathrm{S}}=2 \mathrm{~mm}$ (top), $5 \mu \mathrm{m}$ (bottom), $\mathrm{C}_{\mathrm{C}}=2 \mathrm{~mm}, \Delta \mathrm{E}=3$ $\mathrm{eV}$, DTEM brightness $3 \times 10^{7} \mathrm{~A} \cdot \mathrm{cm}^{-2} \cdot$ steradian $^{-1}$, FEG brightness $2 \times 10^{9} \mathrm{~A} \cdot \mathrm{cm}^{-2} \cdot$ steradian $^{-1}, f_{\text {Rose }}=5$, $\mathrm{f}_{\text {coherence }}=1, \alpha_{\text {HAADF }}=0.01, f_{\text {collected }}=0.2$. These curves are for ideal samples with $100 \%$ contrast; the curves for real samples will be shifted somewhat to the right. 
From Figure 2 we may conclude a number of things, keeping in mind that the models employed are intended for rough estimates only. First, the current DTEM at LLNL should be capable of incoherent imaging on the scale of $10 \mathrm{~ns}$ and a few $\mathrm{nm}$, with the use of an HAADF aperture and an ideal 100\%-contrast sample. At present, the DTEM reaches better than $10 \mathrm{~nm}$ resolution in $15 \mathrm{~ns}$ exposures in conventional mass-thickness (incoherent) bright-field imaging [42], with real samples that inevitably have less than $100 \%$ contrast, so the calculations seem to be reasonably close to reality in this indirect comparison. Extending the DTEM's pulse duration into the $\mu$ s regime should enable coherent imaging modes, including some phase contrast imaging at resolutions near $0.3 \mathrm{~nm}$. Increasing the brightness to be comparable to that of a field emission gun (FEG) would enable nearly the same performance at 10 ns as would be possible with the current brightness at $1 \mu \mathrm{s}$, while the FEG brightness at $1 \mu$ s should be capable of atomic resolution imaging over a wide spatial bandwidth. $\mathrm{C}_{S}$ correction (bottom graph of Figure 3) would allow all three imaging modes to push down to angstrom-scale resolution, where the chromatic aberration becomes the limit according to the present models. Values below $0.1 \mathrm{~nm}$ were not calculated due to the breakdown of the approximations in the spatial coherence envelope calculation, since the convergence angles at the coherence-matched condition become very large near this point. Also, we have neglected electron-electron scattering effects in the imaging lens system, which previous calculations [43] indicate may be the dominant resolution limit in this regime.

The addition of a phase plate (which, for small convergence angles, allows the user to swap the coherent amplitude and phase contrast transfer functions) to an aberration-corrected, high-brightness system could in principle enable atomic resolution at the scale of $10 \mathrm{~ns}$, provided the electron-electron scatter can be minimized. Electron-electron scatter may turn out to be a more serious problem than chromatic aberration, which can be either minimized at the source (by reducing the energy spread of the photoemitted electrons) or corrected in the imaging system. It may be that a polarized electron source could help with the electronelectron scattering problem, but at present this concept is quite speculative. A more direct method is to go to much higher accelerating voltages [28]. This may have disadvantages in terms of radiation damage to the sample and the difficulty of lens engineering, but it may be just about the only way to achieve single-shot near-atomic resolution in sub-nanosecond electron pulses.

\section{Instrumentation}

The current DTEM at LLNL is built on the JEOL 2000FX microscope platform (schematic of the main components is shown in Figure 3 ). The electron optical column has been modified to provide laser access to the photocathode and specimen. A brass drift section has been added between the gun alignment coils and condenser optic that contains a $1^{\prime \prime}$ laser port and $45^{\circ}$ Mo mirror, which directs an on-axis $211 \mathrm{~nm}$ laser pulse towards a $825 \mu \mathrm{m}$ Ta disk photocathode. The 12ns UV laser pulse photoexcites a 15ns FWHM electron pulse from the cathode. This pulse is then accelerated through the electron gun and passes through a hole in the Mo laser mirror and into the electron optics of the TEM column. The electron pulse is aligned and illuminates specimens as in standard TEM operation, and thus all imaging modes can be utilized, e.g., bright-field, dark-field, selected area electron diffraction. A critical step in re- 
engineering the TEM for obtaining high current electron pulses in the single-shot mode was adding electron optics and column sections between the accelerator and condenser sections to better couple the photoemitted electron pulse into the condenser electron optics. Specifically, a weak lens has been installed above the Mo laser mirror and brass drift section. The lens provides increased current by focusing the spatially broad pulsed electron beam through the hole in the laser mirror and condenser system entrance apertures. The coupling lens combined with appropriate condenser lens settings and imaging conditions preserves the brightness of the gun and improves beam quality by reducing the aberrations that can result from a spatially broad electron pulse and high-angle, off-axis electrons.

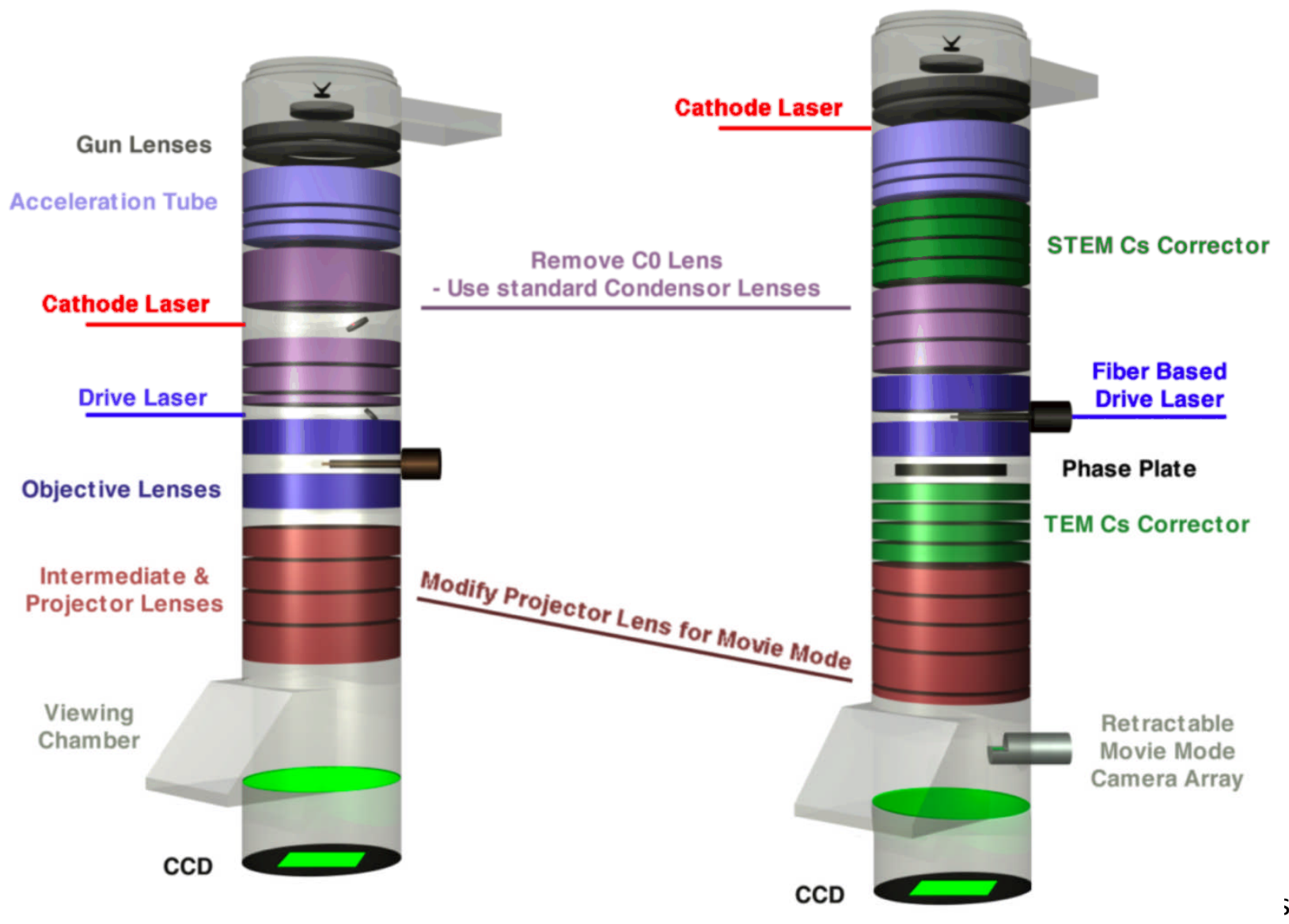

(right) should improve the performance of the microscope significantly. Furthermore, the flexibility of the optics should also allow for the installation of deflection plates and cameras to obtain multiple images for a single specimen drive event (this is also being incorporated on the LLNL DTEM) and a laser based phase plate.

Time-resolved experiments in the DTEM are conducted by first initiating a transient state in the sample and then taking a snap-shot of the transient process with the $15 \mathrm{~ns}$ electron pulse at some preferred time delay after the initiation. In most DTEM experiments, the transient process is initiated with a second laser pulse that enters the TEM column through a modified high-angle X-ray port. For nanosecond time scale experiments, Neodymium doped YAG lasers with pulse duration from 3 to $25 \mathrm{~ns}$ are used that can produce fluences up to $1500 \mathrm{~J} \mathrm{~cm}^{-2}$ on the 
specimen, which is high enough to turn most specimens into a plasma. Thus, by controlling the laser energy and spot on the sample, wide ranges temperatures in the sample and sample heating rates can be produced. The fundamental wavelength $(1064 \mathrm{~nm})$ of these lasers can be frequency converted using nonlinear harmonic generation crystals, e.g., doubled (532 $\mathrm{nm}$ ), tripled $(355 \mathrm{~nm})$ or quadrupled $(266 \mathrm{~nm})$, as dictated by the absorption characteristics of the sample and the desired experimental conditions. For instance, metals have broadband absorption and, thus, all of these wavelengths can be used, while certain semiconductors only absorb sufficient amounts of laser energy in the visible or UV range and may require frequency doubled or tripled laser pulses.

One type of dynamic reaction that has been studied by the DTEM at LLNL involves Reactive Multilayer Foils (RMLFs) which react exothermically to produce intermetallic compounds through self-propagating high-temperature synthesis (SHS). The heat released from mixing chemically-distinct layers is the main driving force in their propagation, which can occur at speeds reaching $40 \mathrm{~m} / \mathrm{s}$ [41]. Until the advent of the DTEM [42-44], most knowledge of SHS details came from post mortem analysis of quenched reactions or DSC slow-heating experiments [45]. Neither one represents the SHS although for different reasons. The former because it leaves voids in the knowledge of the phase evolution during the reaction and the latter because slow heating produces intermediate phases per the phase diagram different from those present during SHS propagation.
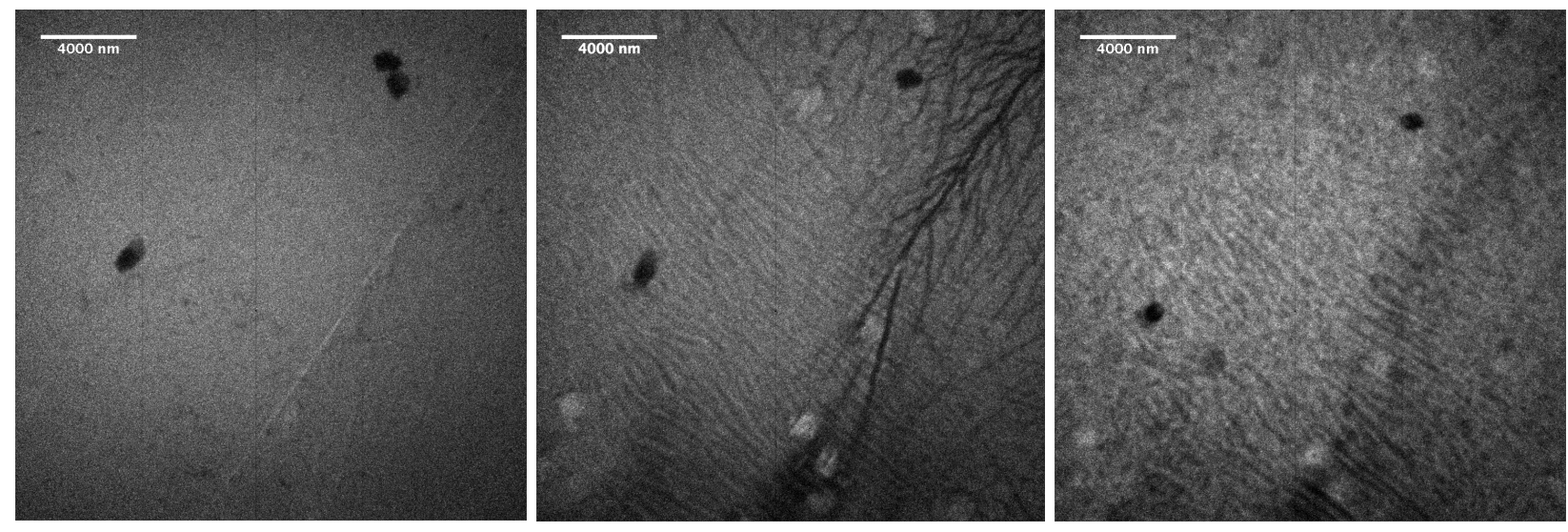

Figure 4: Al-Co multilayer foils in a 1.38:1 atomic percent ratio (a) before propagation, as grown (b) $25 \mu$ s after laser initiation, the spin-like vein of unsteady propagation is captured just prior to quenching (c) microstructure after propagation

In one particular DTEM study, AICo multilayers grown at Sandia National Laboratory were reacted in the DTEM. These layers were grown with 1.38:1 atomic percent Al:Co, with each bilayer measuring $20 \mathrm{~nm}$ and the total foil thickness containing 8 bilayers. The laser initiation caused limited propagation as the conductive and radiative heat loses quenched the reaction prematurely. In Al-Co multilayers, unsteady propagation and spin reaction mode have been observed using optical methods or scanning probe microscopy $[46,47]$. The spin-like and rippled appearance is a manifestation of an oscillatory thermal wave and develops after the balance of heat production and heat diffusion is disturbed $[46,47]$. Figure 4 shows the spin-like 
portion of the propagation front at the tail-end of propagation shortly before quenching occurred. Initially, the laser pre-heats a significant portion of the foil to allow steady-state propagation. Then as thermal diffusion and radiative heat loss overtakes the heat production, oscillations begin and the speed becomes unsteady and slows significantly from that observed for steady state.

To improve the performance of the microscope (i.e. implement the control over the beam described in section 2), several advances in electron optics are being implemented in a new DTEM at UC-Davis. The new DTEM uses the JEOL 2200 double aberration corrected TEM with in-column filter as its base. This microscope implements the photoemission gun within the framework of the field emission gun (note that it is not operating as a field emission gun). This facility means that the inherent gun lens in the system can reproduce the effects of the weak lens implemented in the LLNL instrument without modifying the column. Additionally, the laser can be incorporated into the gun through the standard viewing window in the gun. Both of these factors increase the coupling from the gun to the column and reduce the alignment steps involved in using the DTEM. To further maximize the current and the spatial coherence in the illumination on the specimen, the pre-specimen objective lens is spherical aberration corrected. Correcting for spherical aberration should reduce incoherent broadening of the contrast transfer function and accentuate phase contrast from small signals, making them attractive, for example, for use in liquid cell biological imaging (see next section). It also allows the use of larger aperture and convergence angles at the specimen, thereby increasing the dose without significant loss in beam coherence. To ensure that the maximum contrast is maintained after the beam has interacted with the specimen, the post specimen objective lens is also aberration corrected. In this case, the lens should be optimally corrected for both spherical and chromatic aberrations (the instrument being installed at UC-Davis is only spherical aberration corrected after the specimen at this time). A further advantage for imaging in this system is that the incolumn filter can be used to either filter out inelastically scattered electrons or to use inelastically scattered electrons to form energy filtered images (this gives compositional information to the fast images).

\section{In-situ Stages}

One of the advantages of the DTEM approach is the ability to study materials dynamics insitu in the microscope. To make this method as widely applicable as possible it is important to be able to study materials in environments beyond the high vacuum conditions in the microscope column. In-situ monitoring of materials under variable conditions of temperature and pressure can be achieved in the DTEM using a gas stage. The gas stage that has been designed for the DTEM combines a gas-flow assembly with a modified TEM holder to allow a controlled mixture of up to four gases to be circulated over a specimen. The TEM holder incorporates a window-type environmental cell to contain the desired gaseous environment. Heating of the specimen to create a reaction is then accomplished with the DTEM laser. Reactions can then be controlled by the gas partial pressure within the stage to ensure that interactions occur on the desired timescale for imaging and diffraction. The environmental cell of the TEM holder that has been implemented both at UC-Davis and LLNL was designed in collaboration with Fischione Instruments and accepts a standard 3-mm TEM specimen. The gas 
path length in the environmental cell is currently $250 \mu \mathrm{m}$. The membrane material of the windows is typically amorphous silicon nitride, but can be varied according to experimental needs. The gas flow rate can be adjusted with the flow controllers from $0-5 \mathrm{sccm}$, and gas flow through the cell is achieved via inlet (connected to the flow assembly) and outlet (connected to a turbomolecular pump) tubes that run the length of the holder. Pairing the DTEM with this gas stage creates a unique in-situ characterization tool capable of dynamic studies with exceptional spatio-temporal resolution. Figure 4 shows an atomic-resolution image of a gold nanoparticle, obtained in the gas stage through two $50-\mathrm{nm}$ silicon nitride windows (this result was obtained on a conventional microscope to show the expected resolution when the new UC-Davis DTEM is implemented). This spatial resolution, combined with the temporal resolution of the DTEM, will allow direct, time-resolved observations of gas-solid interactions during, for example, nanoscale catalytic processes. It can provide insight into the synthesis and properties of nanostructures, as well as basic materials properties across multiple spatial and time scales.

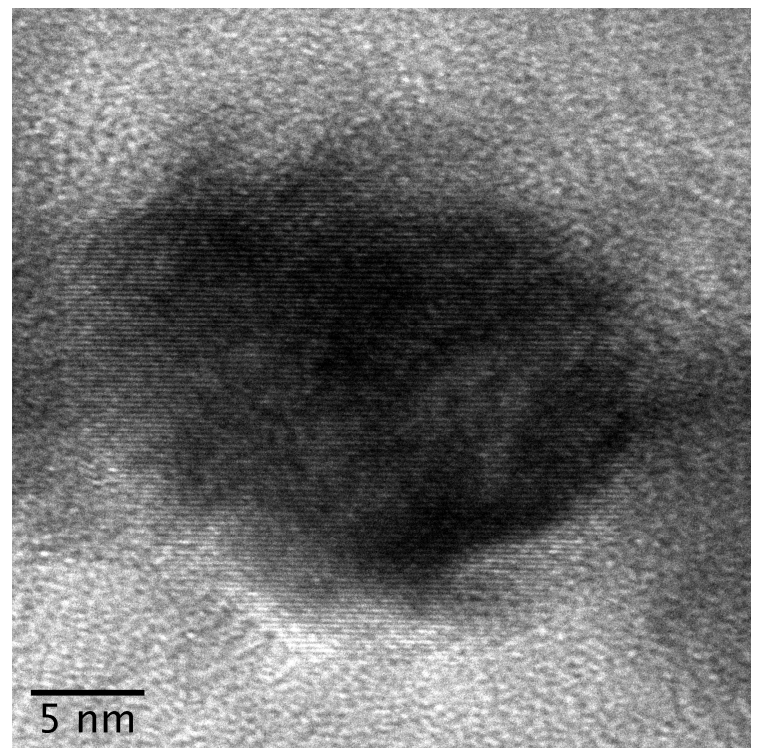

Figure 4: $\quad$ Atomic-resolution, brightfield TEM image of a gold nanoparticle, obtained in the gas stage through two 50$\mathrm{nm}$ silicon nitride windows.

The combination of high temporal and spatial resolution of the DTEM is also highly beneficial in the application of fluid environmental imaging. Capturing images of reactions and free flowing materials is currently encumbered by Brownian motion and mobility from charging effects caused by the incident electron beam. The DTEM is able to overcome these obstacles by capturing images with temporal resolutions of 10 - 1,000 nanoseconds while also permitting low dose imaging conditions to decrease the beam effects on the sample. Therefore experiments which have already demonstrated electrochemical reactions [48], nanoparticle growth [49], and the observation of whole cells [50,51] could benefit from these improved imaging conditions. Conventional TEM imaging resulted in the production of gas bubbles following radiolysis damage to the aqueous environment when viewing whole $E$. coli cells in situ [50]. However, the pulsed imaging mode of the DTEM utilizes doses that are considerably below that for a conventional TEM and should help mitigate beam-induced damage to the fluid environment.

The design of the in situ fluid holder (manufactured by Hummingbird) is based on two thin film electron transparent membranes which encase the solution from the vacuum of the 
electron microscope. These thin films are usually composed of amorphous silicon oxide or silicon nitride with membrane thicknesses ranging from $25-100 \mathrm{~nm}$. The membranes are supported by a $2.6 \times 2.6 \mathrm{~mm}$ silicon support with a window area ranging from 2,500-40,000 $\mu \mathrm{m}^{2}$. Spacing between the windows can be achieved by patterning a support design onto the window surface during fabrication $[48,49]$, depositing an inert material onto the corners of the window surface [50] or by allowing an epoxy to bridge the gap [51]. The resulting thickness of the solution, or fluid path length, is determined by the height of the spacers which range from $50 \mathrm{~nm}$ to several micrometers tall. The use of glue to completely enclose the sample in a vacuum tight environmental chamber has the advantage of allowing analysis within a nondedicated fluid holder, although the reactivity of the glue with the solution, process of polymerization and outgassing, as well as large fluid path lengths can create a significant disadvantage for certain experiments.

The assembly of a continuous flow fluid cell for a dedicated holder is designed to contain the solution by compressing the cell and associated o-rings to create a seal against the vacuum of the microscope. At the same time, the system is open to flow at ambient pressure using microfluidic pumping [52]. The loading procedure for this type of holder consists of a bottom window that acts as a base platform with the membrane side facing upwards. This window is typically loaded with the spacers deposited at the corners and a droplet of the experimental solution placed on the membrane window surface. The top window is loaded with the membrane surface facing down towards the droplet. O-rings positioned below the bottom window and above the top window on the Si substrate are centered about the thin film region while a third o-ring creates a perimeter about the fluid well to isolate the entire chamber from the vacuum of the microscope. Therefore the solution is completely contained within the interior confines of the holder. Flow rates and volumes may be predetermined to supply fresh materials or introduce reactant solutions into the field of view during imaging within the microscope. Future developments of the stage will allow for thermal regulation and electrochemical control.
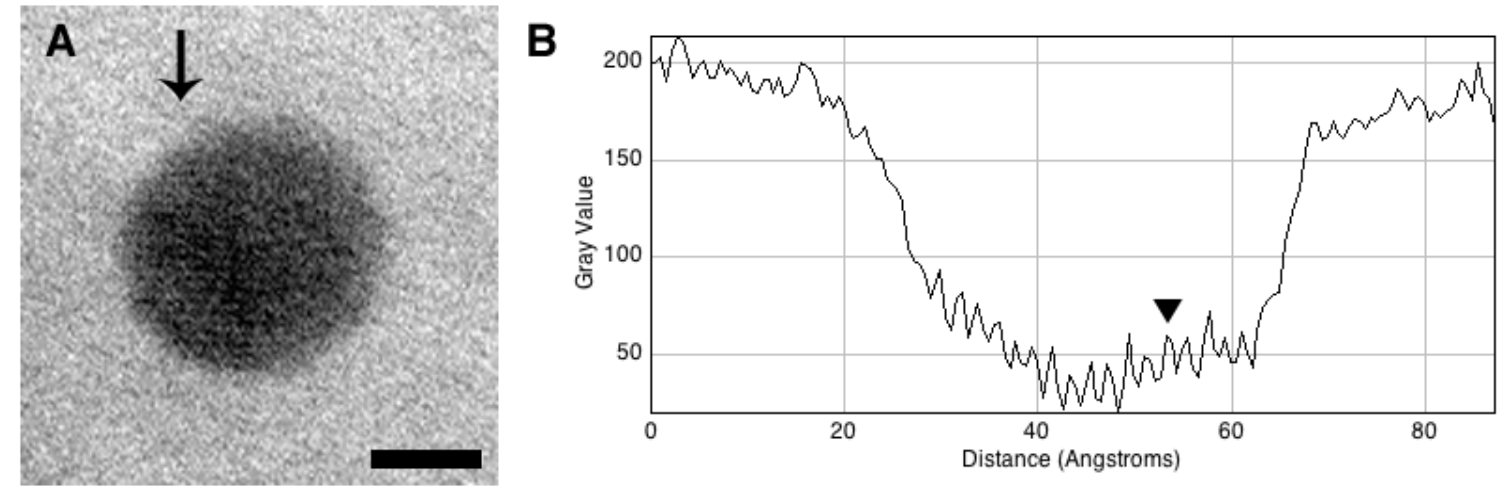

Figure 5: Atomic resolution in situ liquid imaging of nanoparticles. (a) Lead sulfide nanoparticle showing 2 angstrom fringes in Bright Field Scanning TEM. The fluid path length is nominally 50 $\mathrm{nm}$ enclosed by 2 silicon nitride membranes of $50 \mathrm{~nm}$ thickness each. (b) Vertical linescan of the particle in $(A)$ in the direction of the black arrow and a line thickness of 10 pixels. The 2 angstrom spacing can clearly be seen as indicated by the black arrowhead. 
The continuous flow in situ fluid holder described early could be combined with the DTEM to provide a very promising platform for potential studies of colloids, electrochemical growth and biological structure analysis. We have tested such a fluid holder with scanning TEM to image colloidal particles in an effort to reduce the continuous dose over the imaging region. Within a spherical aberration corrected JEM-2200-CS (S)TEM, we were able to demonstrate $2 \AA$ resolution for a lead sulfide nanoparticle with visible lattice fringes (Figure 5). The total sample thickness was nominally $150 \mathrm{~nm}(50 \mathrm{~nm}$ for each silicon nitride membrane and $50 \mathrm{~nm}$ fluid solution). To further improve the attainable spatial resolution either the fluid path length or the membrane thickness will need to be decreased

\section{Summary}

The use of a laser to stimulate photoemission has been shown to provide the ability to create short pulses of electrons in the TEM to study the dynamics in materials and biological systems with nanometer and nanosecond resolution in single-shot mode. The DTEM that is currently operational uses the most basic electron optical components and can be readily upgraded to improve the overall combined spatio-temporal performance - these upgrades are already underway. By incorporating in-situ stages into the microscope, dynamic processes under widely varying environmental conditions can also be studied. In this case, the ability to control the beam and the stimulus to the specimen through a laser will provide unprecedented control and reproducibility to experiments that is not afforded by conventional microscopes and heating stages.

\section{Acknowledgments}

Aspects of this work were performed under the auspices of the U.S. Department of Energy by Lawrence Livermore National Laboratory and supported by the Office of Science, Office of Basic Energy Sciences, Division of Materials Sciences and Engineering, of the U.S. Department of Energy under Contract DE-AC52-07NA27344. Aspects of this work were also supported by DOE NNSA-SSAA grant number DE-FG52-06NA26213, by NIH grant number RR025032-01, by NSF grant and by ExxonMobil. 


\section{References}

[1] M. Haider, S. Uhlemann, E. Schwan, H. Rose, B. Kabius, K. Urban, Nature 392, 768-769 (1998)

[2] P. E. Batson, N. Dellby, O. L. Krivanek, Nature 418, 617-620 (2002)

[3] R. Erni, M. D. Rossell, C. Kisielowski, U. Dahmen, Physical Review Letters 102, 096101 (2009)

[4] K. Kimoto, T. Asaka, T. Nagai, M. Saito, Y. Matsui, K. Ishizuka, Nature 450, 702-704 (2007)

[5] V. Ortalan, A. Uzun, B. C. Gates and N. D. Browning, Nature Nanotechnology 5, 843-847 (2010)

[6] R. Sharma, Journal of Materials Research 20, 1695 (2005)

[7] A. M. Minor, S. A. S. Asif, Z. W. Shan, E. A. Stach, E. Cyrankowski, T. J. Wyrobek, and O. L. Warren, Nature Materials 5 , 697 (2006)

[8] Z. W. Shan, R. K. Mishra, S. A. S. Asif, O. L. Warren, and A. M. Minor, Nature Materials 7, 115 (2008)

[9] B. G. Clark, I. M. Robertson, L. M. Dougherty, D. C. Ahn, and P. Sofronis, Journal of Materials Research 20, 1792 (2005)

[10] A. Radisic, P.M. Vereecken, J.B. Hannon, P.C. Searson, and F.M. Ross, Nano Letters 6, 238-242 (2006)

[11] H. D. Espinosa, Y. Zhu, N. Moldovan, J. Microelectromechanical Systems 16, 1219-1231 (2007)

[12] P. L. Gai, Topics in Catalysis 21, 161-173 (2002).

[13] W. Q. Han, L. J. Wu, Y. M. Zhu, M. Strongin, Nano Letters 5, 1419-1422 (2005).

[14] K. H. Min, R. Sinclair, I. S. Park, S. T. Kim, U. I. Chung, Phil. Mag 85, 2049-2063 (2005).

[15] J. M. Howe. and H. Saka, MRS Bulletin 29 951-957 (2004)

[16] A. Loveridge-Smith, A. Allen, J. Belak, T. Boehly, A. Hauer, B. Holian, D. Kalantar, G. Kyrala, R. W. Lee, P. Lomdahl, M. A. Meyers, D. Paisley, S. Pollaine, B. Remington, D. C. Swift, S. Weber, and J. S. Wark, Phys. Rev. Lett. 86, 2349(2001)

[17] D. H. Kalantar, J. F. Belak, G. W. Collins, J. D. Colvin, H. M. Davies, J. H. Eggert, T. C. Germann, J. Hawreliak, B. L. Holian, K. Kadau, P. S. Lomdahl, H. E. Lorenzana, M. A. Meyers, K. Rosolankova, M. S. Schneider, J. Sheppard, J. S. Stolken, J. S. Wark, Phys. Rev. Let. , 95, 075502/1-4 (2005)

[18] J. A. Hawreliak, D. H. Kalantar, J. S. Stolken, B. A. Remington, H. E. Lorenzana, J. S. Wark, Phys. Rev. B 78, 220101 (2008)

[19] J. Spence and M. R. Howells, Ultramicroscopy 93, 213-222 (2002).

[20] A. H. Zewail, Annu. Rev. Phys. Chem. 2006, 57, 65-103 
[21] A. H. Zewail, J. Phys Chem A 2000, 104, 5660-5694

[22] B. J. Siwick, J. R. Dwyer, R. E. Jordan, R. J. D. Miller, Science 2003, 302, 1382-1385

[23] J. Cao, Z. Hao, H. Park, C. Tao, D. Kau, and L. Blaszczyk, Appl Phys. Letts. 83, 1044-1046 (2003)

[24] D. J. Masiel, T. LaGrange, B. W. Reed, T. Guo and N. D. Browning, ChemPhysChem11, 2088-2090 (2010)

[25] M. L. Taheri, S. McGowan, L. Nikolova, J. E. Evans, N. Teslich, J. P. Lu, T. LaGrange, F. Rosei, B. J. Siwick, and N. D. Browning, Applied Physics Letters 97, 032102 (2010)

[26] Thomas LaGrange, David S. Grummon, Nigel D. Browning, Wayne E. King, and G. H. Campbell, Applied Physics Letters 94, 184101 (2009)

[27] M. L. Taheri, B. W. Reed, T. B. Lagrange \& N. D. Browning, Small 4, 2187-2190 (2008)

[28] M. R. Armstrong, N. D. Browning, B. W. Reed, B. R. Torralva, Applied Phys Letts 90, 114101 (2007)

[29] Michael Armstrong, Ken Boyden, Nigel D. Browning, Geoffrey H. Campbell, Jeffrey D. Colvin, Bill DeHope, Alan M. Frank, David J. Gibson, Fred Hartemann, Judy S. Kim, Wayne E. King, Thomas B. LaGrange, Ben J. Pyke, Bryan W. Reed, Richard M. Shuttlesworth, Brent C. Stuart, Ben R. Torralva, Ultramicroscopy107, 356-367 (2007)

[30] Thomas B. LaGrange, Michael Armstrong, Ken Boyden, Curtis Brown, Nigel D. Browning, Geoffrey H. Campbell, Jeffrey D. Colvin, Bill DeHope, Alan M. Frank, David J. Gibson, Fred Hartemann, Judy S. Kim, Wayne E. King, Ben J. Pyke, Bryan W. Reed, Richard M. Shuttlesworth, Brent C. Stuart, Ben R. Torralva, Applied Physics Letters 89, 044105 (2006)

[31] O. Bostonjoglo and R. Leidtke, Advances in Imaging and Electron Physics 60, 451 (1980).

[32] O. Bostonjoglo, R. Elschner, Z. mao, T. Nink, M. Weingartner Ultramicroscopy 81, 141 (2000).

[33] O. Bostonjoglo, Advances in Imaging and Electron Physics 121, 1 (2002).

[34] B. Barwick, H. S. Park, O. H. Kwon, J. S. Baskin, A. H. Zewail, Science 2008, 322, 12271231

[35] F. Carbone, O. H. Kwon, A. H. Zewail, Science 2009, 325, 181-184

[36] B. W. Reed, T. LaGrange, R. M. Shuttlesworth, D. J. Gibson, G. H. Campbell, and N. D. Browning, Review of Scientific Instruments81, 053706 (2010)

[37] B. W. Reed, M. R. Armstrong, N. D. Browning, G. H. Campbell, J. E. Evans, T. B. LaGrange, and D. J. Masiel, Microscopy and Microanalysis15, 272-281 (2009)

[38] W. E. King, G. H. Campbell, A. Frank, B. Reed, J. F. Schmerge, B. J. Siwick, B. C. Stuart \& P. M. Weber, J Appl Phys 97, 111101 (2005)

[39] O. L. Krivanek, In High-Resolution Transmission Electron Microscopy and Associated Techniques, Buseck, P., Cowley, J. \& Eyring, L. (Eds.), pp. 519-567. New York: Oxford University Press (1992) 
[40] A. Rose, In Advances in Electronics and Electron Physics, Marston (Ed.), New York: Academic Press (1948)

[41] J. P. McDonald, M. A. Rodriguez, E. D. Jones, Jr., and D. P. Adams, J. Mater. Res. 25(4): 718-727 (2010).

[42] J. S Kim, T Lagrange, B. W Reed, M. L Taheri, M. R Armstrong, W. E King, N. D Browning, G. H Campbell, Science 321: 1472 (2008).

[43] T. LaGrange, G.H. Campbell, B.W. Reed, M. L. Tehari, J.B. Pesavento, J.S. Kim, N.D. Browning, Ultramicroscopy, 108 [11] 1441 - 1449 (2008).

[44] J. S. Kim, T. LaGrange, B. W. Reed, R. Knepper, T. P. Weihs, N. D. Browning, G. H. Campbell, Acta Materialia 593571 - 3580 (2011).

[45] C Michaelsen, K Barmak and T P Weihs, J. Phys. D: Appl. Phys. 30:3167-3186 (1997).

[46] D. Aurongzeb, Appl. Phys. Lett. 92(14):141914 (2008).

[47] J. P. McDonald, V. C. Hodges, E.D. Jones, Jr., and D. P. Adams, Appl. Phys. Lett. 94(3): 034102 (2009).

[48] M. J. Williamson, R. M. Tromp, P. M. Vereecken, R. Hull, and F. M. Ross, Nature Mater. 2, 532-536 (2003).

[49] H. Zheng, R. K. Smith, Y. Jun, C. Kisielowski, U. Dahmen, and A. P. Alivisatos, Science 324, 1309-1312 (2009).

[50] K. L. Liu, C. C. Wu, Y. J. Huang, H. L. Pang, H. Y. Chang, P. Chang, L. Hsu, and T. R. Yew, The Royal Society of Chemistry 8, 1915-1921 (2008).

[51] N. de Jonge, D. B. Peckys, G. J. Kremers, and D. W. Piston, PNAS 106 (7), 2159-2164 (2008).

[52] E. A. Ring and N. de Jonge, Microscopy and Microanalysis 16, 622-629 (2010). 